\title{
Dynamical transitions and sliding friction in the two-dimensional Frenkel-Kontorova model
}

\author{
Enzo Granato \\ Laboratório Associado de Sensores e Materiais, Instituto Nacional de Pesquisas Espaciais, 12201 São José dos Campos, SP, Brazil \\ S. C. Ying \\ Department of Physics, Brown University, Providence, Rhode Island 02912
}

(Received 10 August 1998; revised manuscript received 8 October 1998)

\begin{abstract}
The nonlinear response of an adsorbed layer on a periodic substrate to an external force is studied via a two-dimensional uniaxial Frenkel-Kontorova model. The nonequlibrium properties of the model are simulated by Brownian molecular dynamics. Dynamical phase transitions between pinned solid, sliding commensurate and incommensurate solids and hysteresis effects are found that are qualitatively similar to the results for a Lennard-Jones model, thus demonstrating the universal nature of these features. [S0163-1829(99)04707-4]
\end{abstract}

\section{INTRODUCTION}

In recent years, there has been an increasing effort aimed at trying to understand the frictional force between two macroscopic sliding surfaces at the microscopic level. ${ }^{1-9}$ It is now understood that in the case of boundary lubrication, the tightly bound monolayer in the regions of closest contact between the two surfaces plays a crucial role in determining the macroscopic sliding friction. At the same time, new experimental techniques such as the quartz-crystal microbalance $^{10}(\mathrm{QCM})$ and atomic force microscope ${ }^{3}$ (AFM) allow the direct probing of frictional force at the atomic level. Thus, the central theoretical issue in understanding boundary lubrication and atomic-scale friction is to determine how an adsorbed layer responds to an external driving force. In the linear regime, the response is characterized by the collective diffusion constant, which is itself an important transport coefficient in determining surface dynamics such as growth of thin films. ${ }^{11}$ The nonlinear regime is the one relevant for actual conditions of macroscopic sliding motion. Persson $^{2}$ has made a series of pioneering studies on this problem based on a model of an adlayer of particles interacting with Lennard-Jones potentials. The central result is that there are intriguing dynamic phase transitions between pinned solid, sliding solid, and liquid phases. Strong hysteresis effects exist in the transitions between these phases, leading to the "stick and slip" motion often observed in the sliding motion between two macroscopic flat surfaces under an external driving spring. In addition, Persson has used a general hydrodynamic argument to predict that the ratio of the static and sliding friction threshold should take a universal value of approximately 2 for all systems with strong coupling to the substrate.

The qualitative features of the results from this isotropic Lennard-Jones system are very encouraging. Besides the relevance for the boundary lubrication problem, the dynamic phase transitions that have been observed are of intrinsic interest and very different from the more familiar equilibrium transitions. However, actual adsorbate layers usually have strong anisotropies and complicated many-body interactions among the adatoms. The question then arises as to how universal are the results for the Lennard-Jones system.
Recently, Braun et al. ${ }^{7}$ have studied the nonlinear mobility of an adlayer based on a generalized vector FrenkelKontorova model. They have also observed a strong hysteresis effect in the transition between the pinned and running state, while the nature of the running state and the ratio of the static to sliding friction threshold they obtained are quite different from the corresponding results of Persson. However, it is difficult to make a direct comparison between these studies. Persson focused mainly on an initial pinned state that is commensurate with the substrate, while Braun et al. focus almost exclusively on an initial state that is incommensurate with the substrate. In the latter case, the sliding motion is dominated by the response of the kinks or antikinks to the external force.

To facilitate a more direct comparison with Persson's study and address the question of universality in the salient features, we present in this paper the results from a study based on a uniaxial Frenkel-Kontorova model that is different from the one employed by Braun et al. In particular, the particles in our model have harmonic springlike interactions that prevent them from moving away from each other. This implies a fixed coordination number for each adparticle and a very different nature of the liquid phase from the corresponding phase for the Lennard-Jones system. Moreover, the particle displacement is a scalar variable in contrast to the vector displacements in the other models. Also, in this paper we focus mainly on an initial pinned state that is commensurate with the substrate. Surprisingly, we find that almost all the features of the dynamic phase transitions and hysteresis effects found in the Lennard-Jones system are present also for our generalized Frenkel-Kontorova model. The only notable difference between the models is in the value of the ratio of the static to sliding friction threshold. We present below the details of the model and the results. Preliminary results have been reported in an earlier conference proceeding. 5

\section{MODEL}

We consider a two-dimensional version of the FrenkelKontorova (FK) model, which is the simplest extension of the one-dimensional model, ${ }^{13}$ obtained by coupling linear harmonic chains along the additional dimension. The particle 


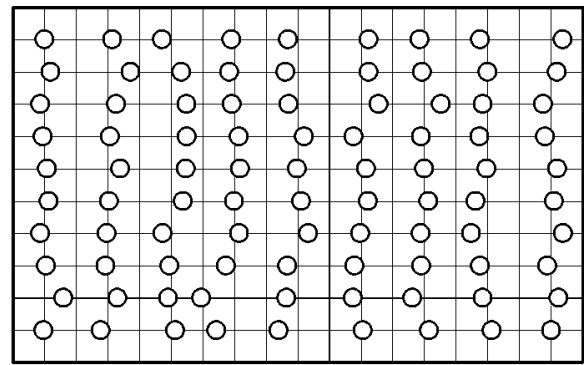

$T>T_{0}$

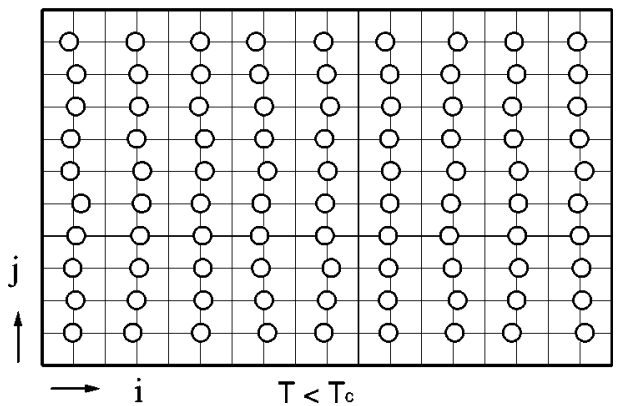

FIG. 1. Snapshot pictures of the adsorbate above and below the depinning temperature $T_{c}$. (a) $T=3$ and (b) $T=0.5$.

displacement is always along the same direction, which makes it particularly convenient for numerical computations. This model is clearly more appropriate to describe uniaxial systems of adsorbed layers; ${ }^{14}$ however, we believe that the main features of the dynamical behavior that we consider in this work should be independent of this type of anisotropy. We have in fact checked that the dynamical transitions and hysteresis effects remain essentially the same if a more isotropic version of the model ${ }^{12}$ is used.

The model is described by the Hamiltonian

$$
\begin{aligned}
H= & \sum_{i, j}\left[\frac{p_{i j}^{2}}{2 m}+\frac{K_{x}}{2}\left(x_{i+1, j}-x_{i j}-b\right)^{2}+\frac{K_{y}}{2}\left(x_{i, j+1}-x_{i j}\right)^{2}\right. \\
& \left.-U_{o} \cos \left(\frac{2 \pi x_{i j}}{a}\right)\right]
\end{aligned}
$$

where $m$ is the mass of the particles; $U_{o}$ the amplitude of the periodic potential with period $a ; K_{x}$ and $K_{y}$ are elastic constants in the $x$ and $y$ directions, and $b$ is the average distance between the particles in the absence of the external potential. The second term in the brackets represents the elastic interaction within a chain and the third term the interaction between adatoms on different chains. Note that the index $i, j$ labels the particles within a chain with integer $i(i$ $\left.=1, \ldots, N_{x}\right)$ and the chain with integer $j\left(j=1, \ldots, N_{y}\right)$, so that the system consists of a total number of $N_{x} \times N_{y}$ particles. In the simulations we have considered $N_{x}=N_{y}$ $=N$ and $K_{x}=K_{y}=K$. Typical equilibrium particle configurations above and below the depinning temperature $T_{c}$ are illustrated in Fig. 1 for $b / a=2$.

We use periodic boundary conditions in most of the calculations. This is obtained by requiring that the particle $\left(N_{x}+1, j\right)$ is a periodic image of the particle $(1, j)$ in the same chain and so $x_{N_{x}+1, j}=x_{1, j}+N_{s} a$, where $N_{s}$ is the num- ber of local minima of the periodic substrate in the $x$ direction. In the other direction, periodic boundary condition means that $x_{i, N_{y}+1}=x_{i, 1}$. The average distance between particles $b$ in each chain is determined through the boundary condition and is given by $b=N_{s} a / N$ and therefore the overlayer coverage, or atomic concentration, can be defined as $\theta=N / N_{s}=a / b$. With this set up, $\theta$ and $b$ cannot be changed independently. With free boundary conditions in the $x$ directions, the ratio $b / a$ can assume arbitrary nonrational values. The system now can remain commensurate for a given $\theta$ only up to a critical value $\delta_{c}$ of the misfit parameter ${ }^{13} \delta$ $=(b-a) / a$. This boundary condition is particularly useful to study incommensurability effects.

An important feature of the model is that the particles have a fixed number of neighbors. So, even at high temperatures, where a liquid phase with particles diffusing around independently is usually expected, there is some crystalline order although the correlations decay as a power law. ${ }^{14} \mathrm{We}$ shall, nevertheless, refer to this phase as a "fluid," keeping in mind its distinct nature. At low temperatures, the periodic potential is able to pin the overlayer and true long-range order prevails leading to a finite static friction. The low- and high-temperature phases are separated by a commensurate solid to fluid transition at a critical temperature $T_{c}$ where the overlayer at high temperatures becomes disordered and is effectively depinned from the substrate. We shall also refer to this transition as "melting" although topological defects, as dislocations, are not allowed due to the fixed-neighbor constraint. In the absence of a periodic potential, disorder plays an important role in pinning the overlayer ${ }^{15,16}$ and glassy behavior is expected.

The periodic potential in Eq. (1) arises from an adiabatic average over the substrate degrees of freedom. The nonadiabatic excitations of the substrate provide energy exchange between the substrate and the adlayer and lead to damping and fluctuation in the motion of the adatoms. In our model, this effect is described by the usual Markovian-Langevin dynamics. The substrate acts as a heat bath at an equilibrium temperature $T$ and the coupling of the substrate excitations and adlayer is characterized by a friction parameter $\eta$. Note that in the absence of the periodic potential, the adatom interactions do not affect the collective response of the adlayer and the macroscopic sliding friction is equal to the microscopic coupling parameter $\eta$.

\section{GENERAL PROPERTIES OF SLIDING FRICTION}

To characterize the response of the adlayer to the external driving force, we define the sliding friction coefficient $\bar{\eta}$ through the relation

$$
v_{d}=F / m \bar{\eta},
$$

where $v_{d}$ is the average velocity of all the adparticles representing the drift velocity in the direction of the force. In general, the sliding friction $\bar{\eta}$ has a complicated dependence on the external force $F$ in the nonlinear regime. First, we remark on a number of qualitative features of the drift velocity $v_{d}$ and the sliding friction $\bar{\eta}$ that can be readily understood without detailed calculations. ${ }^{18}$ In the presence of the external force $F$, the potential is tilted like a washboard with 
local minima which become weaker as the strength of the force is increased. Beyond a critical force value $F_{o}$, the effective potential minima disappears. For the present model, $F_{o}=2 \pi U_{o}$, the same result as for the one-dimensional case. ${ }^{14}$ At $T=0$ and for a commensurate value of coverage $\theta, v_{d}$ remains zero for any applied force less than $F_{o}$. When $F \gg F_{o}$, the layer slides as a whole with a sliding friction $\bar{\eta} \geqslant \eta$. At low but finite temperatures, the drift velocity induced by a small external force is limited by the nucleation and mobility of defects in the commensurate structure. In one dimension, these defects are kinks and antikinks (local compression or extension of the commensurate atomic configuration) and can always be excited at any finite temperature leading to a finite $\bar{\eta}$ at any $T \neq 0$. In two dimensions, however, the corresponding excitations are linear defects (domain walls) that have a nonzero free energy per unit length for temperatures below the melting transition temperature $T_{c}$. As thermal fluctuations, these defects can only be nucleated as closed loops with an activation energy proportional to its length and therefore do not contribute significantly to the linear mobility of the system, $\lim _{F \rightarrow 0} v_{d} / F$, in the thermodynamic limit and one expects that, for a pinned solid, $1 / \bar{\eta}$ is essentially zero for $T<T_{c}$ in this regime. When the external force is increased from zero, activated nucleation and growth of commensurate domains induced by the external force can contribute to a finite mobility, leading to a nonlinear friction $\bar{\eta}>\eta$ with a complicated dependence on the external force $F$. Eventually, in the limit of large external force such that the effect of the periodic potential is negligible altogether, $\bar{\eta}$ again reduces to the microscopic friction parameter $\eta$.

In the nonlinear regime, the behavior of drift velocity $v_{d}$ for increasing external force $F$ is expected to depend strongly on the initial phase ${ }^{2}$ at $F=0$. If the adsorbate, when $F=0$, is a fluid, $v_{d}$ will be nonzero for arbitrarily small external force. This result is also true for the present model. In the fluid phase above $T_{c}$, the overlayer is essentially depinned and any small force leads to nonzero $v_{d}$. Moreover, the relation $v_{d}=f(F)$ should show no hysteresis. For an initial state at $F=0$ that is incommensurate, the critical value of the force for finite mobility can be much lower as shown in previous works of Persson ${ }^{2}$ and Braun et al. ${ }^{7}$

\section{MOLECULAR-DYNAMICS SIMULATION}

To analyze the nonlinear sliding friction of the model presented in Sec. II, we have performed a molecular-dynamics simulation study of the Langevin equation resulting from the model. An external driving force $F$, representing the effect of the other surface in the boundary lubrication problem, is assumed to act on each of the adsorbates. The equation of motion for the particle with coordinate $x_{i j}$ is given by the Langevin equation,

$$
m \ddot{x}_{i j}+m \eta \dot{x}_{i j}=-\frac{\partial V}{\partial x_{i j}}-\frac{\partial U}{\partial x_{i j}}+F+f_{i j}
$$

where $U$ is the periodic potential, $V$ is the adsorbateadsorbate harmonic interaction potential, and $f_{i j}$ is a random force that is related to the microscopic friction parameter $\eta$ by the fluctuation dissipation theorem

$$
\left\langle f_{i j}(t) f_{i^{\prime} j^{\prime}}\left(t^{\prime}\right)\right\rangle=2 \eta m k_{B} T \quad \delta_{i, i^{\prime}} \delta_{j, j^{\prime}} \delta\left(t-t^{\prime}\right) .
$$

We have studied the sliding friction by simulating the above equations using methods of Brownian molecular dynamics, ${ }^{17}$ which treat accurately the effect of the systematic and stochastic forces in the problem. The calculations were performed at a commensurate coverage $\theta=1 / 2(b / a$ $=2$ ). Typically, the time variable was discretized with time step $\delta t=0.02-0.06 \tau$ where $\tau=\left(\mathrm{ma}^{2} / U_{o}\right)^{1 / 2}$ and $4-10$ $\times 10^{5}$ time steps were used in each calculation allowing $10^{5}$ time steps for equilibration. Calculations were performed as function of temperature and external force on systems containing $N \times N$ adsorbate particles, where typically $N=10$, and the elastic constant was set to $K=10$.

In the calculations, the system was allowed to evolve into a steady state such that the time average of physical quantities, like the drift velocity $v_{d}$, approached a constant. The effective temperature $T^{*}$ of the overlayer during sliding was obtained by two different methods. Using the fluctuation of particle velocities from the average as

$$
k T^{*}=m\left(\left\langle v^{2}\right\rangle-\langle v\rangle^{2}\right)
$$

and by equating the energy transferred to the adsorbed system by the external force to the energy transferred to the substrate, which gives ${ }^{2}$

$$
\frac{T^{*}}{T}=1+\frac{F^{2}}{m \eta \bar{\eta} T}\left(1-\frac{\eta}{\bar{\eta}}\right)
$$

where $\bar{\eta}=F / m v_{d}$. The two methods agree within the estimated errors, for calculations expected to be equilibrated in a steady state, and provide additional support for the assumption that local thermalization of the overlayer can be described by an effective temperature $T^{*}$.

\section{RESULTS AND DISCUSSION}

In this section we present results from our moleculardynamics simulations. We use units in which $a=1, m=1$, and $U_{0}=1$, which sets the scales for length, mass, and energy, respectively. The corresponding time scale is $\tau$ $=\left(m a^{2} / U_{o}\right)^{1 / 2}$, and the temperature scale is $T_{0}=U_{0} / k_{B}$. It is also convenient to normalize the external force by the zero-temperature critical value $F_{o}$.

To obtain an estimate of critical temperature $T_{c}$, we calculated $\bar{\eta}$, as a function of temperature for a small value of the external force, $F / F_{o}=0.035$. At low temperatures, $1 / \bar{\eta}$ is essentially zero and the adlayer is in a pinned state. As the temperature is increased $1 / \bar{\eta}$ remains zero until ${ }^{5} T_{c} \sim 2.3$, beyond which it increases significantly and should approach 1 at high enough $T$. The value of $T_{c}$ correspond to the commensurate solid-fluid transition. Above $T_{c}$, the overlayer is effectively depinned from the substrate.

In Fig. 2 we show the behavior of $v_{d}$ and the effective temperature of the layer $T^{*}$, obtained at a temperature $T$ $=3$ which is above $T_{c}$. In this case, the adlayer is in a fluid state at zero external force. The results for $F$ increasing from 

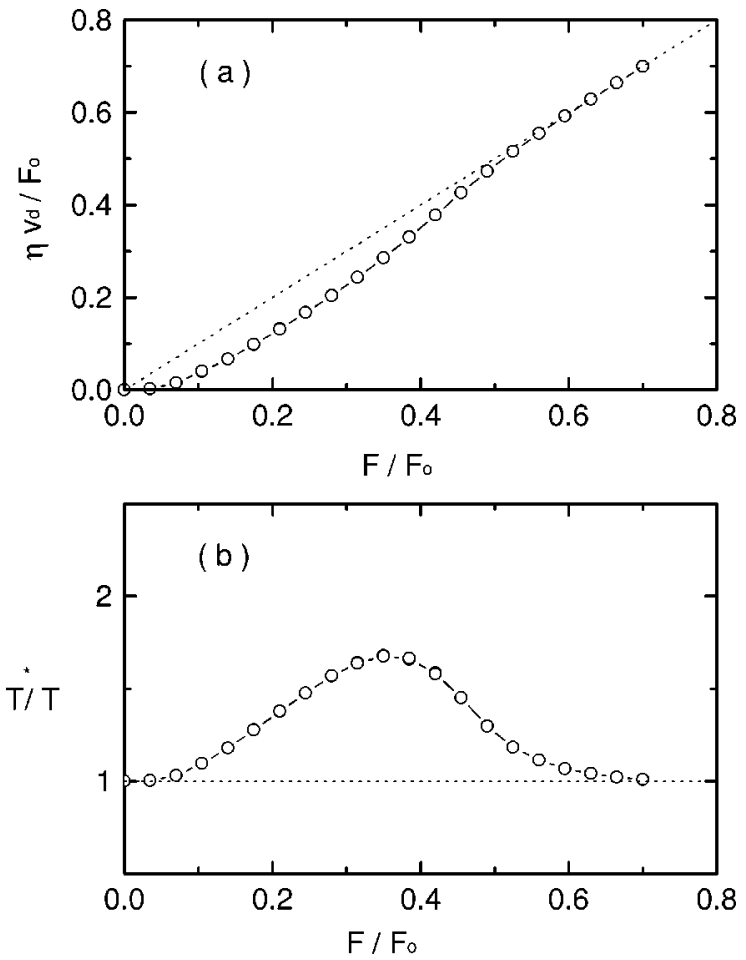

FIG. 2. Drift velocity $v_{d}$ and effective temperature $T^{*}$ as a function of external force $F$ for $\eta=0.6$ and $T=3$. Dotted lines correspond to free overlayer behavior.

zero and decreasing from its highest value are the same, showing a total absence of hysteresis effects. The effective friction coefficient $\bar{\eta}$ is finite even at vanishingly small values of $F$. In the absence of the periodic potential, $\bar{\eta}=\eta$ and $v_{d}$ should depend linearly on $F$ as indicated by the dotted line in Fig. 2. In the present case, the net motion of the overlayer is limited by defect excitations induced by the coupling to the periodic potential leading to an enhancement of $\bar{\eta}$ over the microscopic friction parameter $\eta$ and an increase of $T^{*}$ over the equilibrium temperature $T$, as $F$ increases. The sliding friction $\bar{\eta}$ approaches $\eta$ only for larger $F$. The calculation of the effective temperature $T^{*}$ assumes that the particle velocities have a Maxwellian probability distribution of width $k_{B} T^{*}$. In fact, as shown in Fig. 3 the velocity distribution can be well described by a Gaussian distribution.

For $T<T_{c}$, however, where a commensurate solid phase

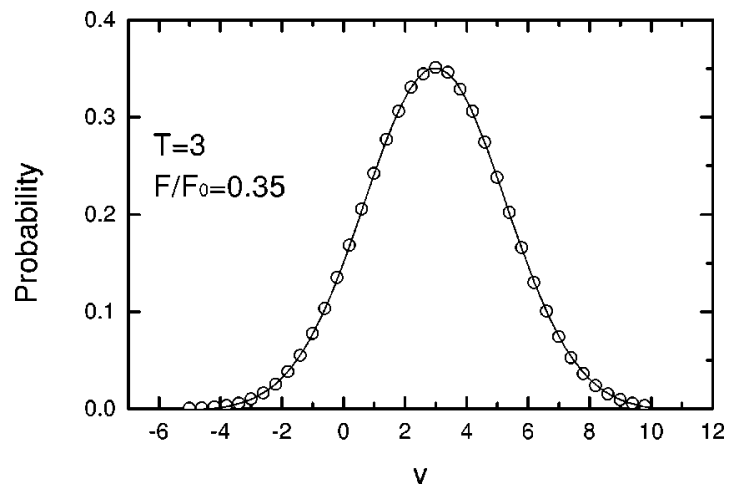

FIG. 3. Probability distribution (circles) of particle velocities in the stead state for $F / F_{o}=0.35$ in Fig. 2. Solid line is a Gaussian fit.
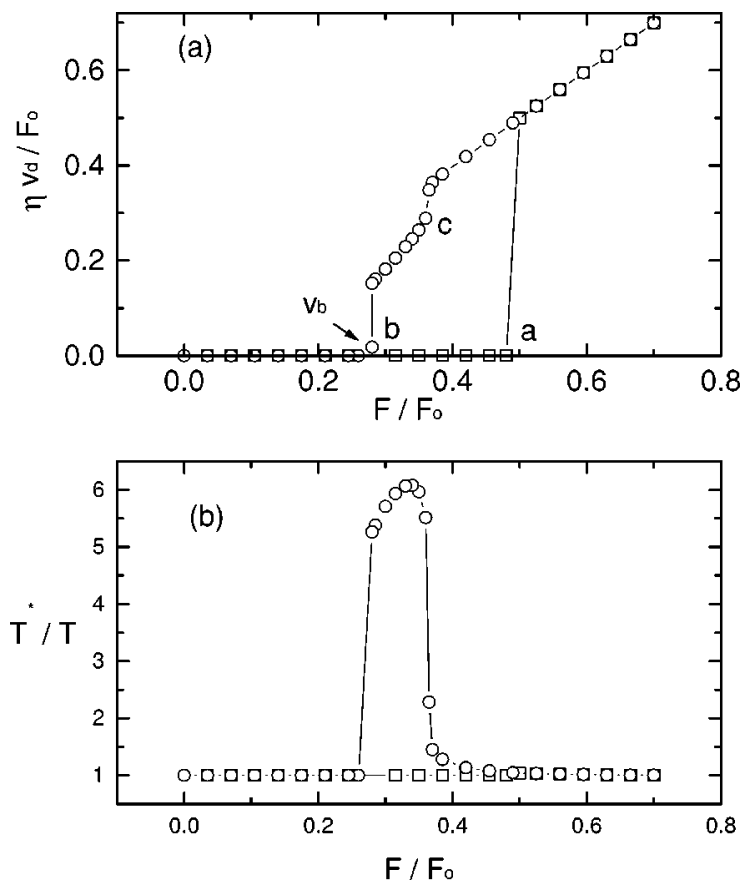

FIG. 4. Drift velocity $v_{d}$ and effective temperature $T^{*}$ as a function of external force $F$ for $\eta=0.6$ and $T=0.5$ and periodic boundary conditions. Squares correspond to data for increasing $F$ and circles for decreasing $F$. The arrow indicates the minimum velocity $v_{b}$.

prevails when $F=0$, a critical force $F_{a}$ is necessary to depin and initiate sliding. It was found for the Lennard-Jones system $^{2}$ that in this case the nonlinear sliding friction exhibits hysteresis as a function of $F$, i.e., the relation between velocity and external force depends on whether $F$ increases from zero or decreases from a high value. We find the same behavior in our model for sufficiently low temperatures. In Fig. 4, we show the results for $v_{d}$ and the effective temperature of the adlayer $T^{*}$ as a function of $F$ for $\eta=0.6$ obtained at a temperature $T=0.5$, which is much less than $T_{c}$. This temperature is also considerably below the amplitude of the periodic potential $U_{o}$. As will be discussed below, we find that $T<U_{0}$ seems to be necessary in order that a sharp hysteresis feature appears in the $v_{d} \times F$ characteristics. The calculation was performed with the adlayer initially in a pinned state, then the external force was increased to different values. An hysteresis loop is clearly seen in Fig. 4 and shares the same features as found in the simulations of the LennardJones systems. $^{2}$

In Fig. 4, it is shown that the initial sliding phase, when the applied force is increased beyond the static threshold $F_{a}$, has almost the same temperature as the substrate and the substrate potential provides no additional resistance. This is a sliding solid phase. It corresponds to the floating or incommensurate solid phase in the equilibrium situation. It is important to note that this phase is dynamically generated by the external field since the initial temperature is below $T_{c}$ and a commensurate solid phase would be the equilibrium state. With the choice of periodic boundary conditions in our model, the misfit $\delta$ is zero and the configuration of the sliding incommensurate solid phase in Fig. 4 is indistinguishable from a commensurate phase. However, using free boundary conditions and varying the misfit parameter we can confirm 

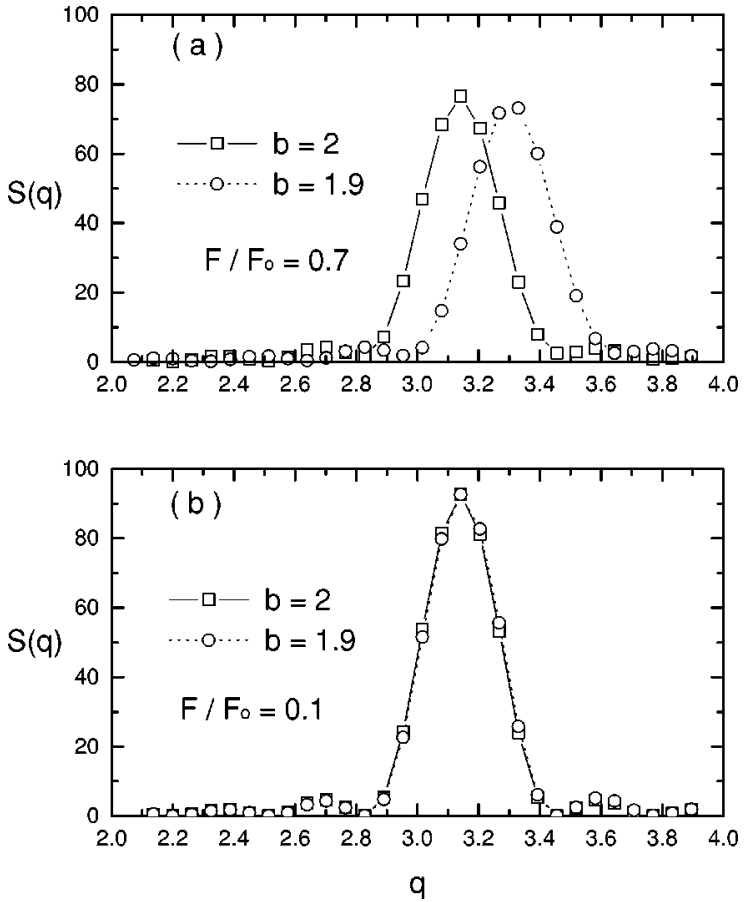

FIG. 5. Structure factor $S(q)$ for $b / a=2$ and $b / a=1.9$ with $\eta$ $=0.6$ and $T=0.5$ and free boundary conditions, in the sliding (a) and pinned (b) phases.

that this phase corresponds to a sliding solid phase incommensurate with the substrate. In Fig. 5 we show the structure factor $S(q)$ for two values of the overlayer lattice spacing, $b / a=2$ and $b / a=1.9$, and at two different limits of the external force, $F / F_{o}=0.1$ in the pinned phase [Fig. 5(b)] and $F / F_{o}=0.7$ in the sliding phase [Fig. 5(a)]. In the sliding phase, $S(q)$ is peaked at the wavevector $2 \pi / b$ independent of the substrate while it remains locked to the commensurate value $\pi$ in the pinned phase. Thus the external force induces a pinned commensurate solid to sliding incommensurate solid transition at $F=F_{a}$. As the force is decreased from its highest value through $F_{a}$, there is a jump in $T^{*}$ accompanied by a simultaneous decrease in $v_{d}$ at a critical force $F_{c}$. This corresponds to an overheating of the overlayer to an effective temperature that is above $T_{c}$, dynamically "melting" the sliding solid phase. This can be regarded as a sliding solid to fluid transition. Finally at $F=F_{b}$, the fluid phase condenses into a pinned commensurate solid phase and the temperature of the adlayer and the substrate become the same again. The fact that there is a condensation from the "melted" phase is confirmed by noting that the temperature of the adlayer at this condensation is almost identical to the equilibrium critical temperature $T_{c}$. Persson ${ }^{2}$ has observed very similar dynamical transitions for Lennard-Jones systems, showing that these features are universal in the nonlinear response of overlayers and not just for specific models. In fact, other versions of the Frenkel-Kontorova model also show some of these features. ${ }^{7}$ In addition the present results show that a conventional "liquid" phase is not required and a high-temperature thermally disordered phase with nonzero linear mobility is sufficient to show some of these effects.

We now consider the behavior of the nonlinear sliding friction at a higher temperature $T=1.5$ but still less than $T_{c}$. As can be seen from Fig. 6, the hysteresis disappears but $F_{a}$
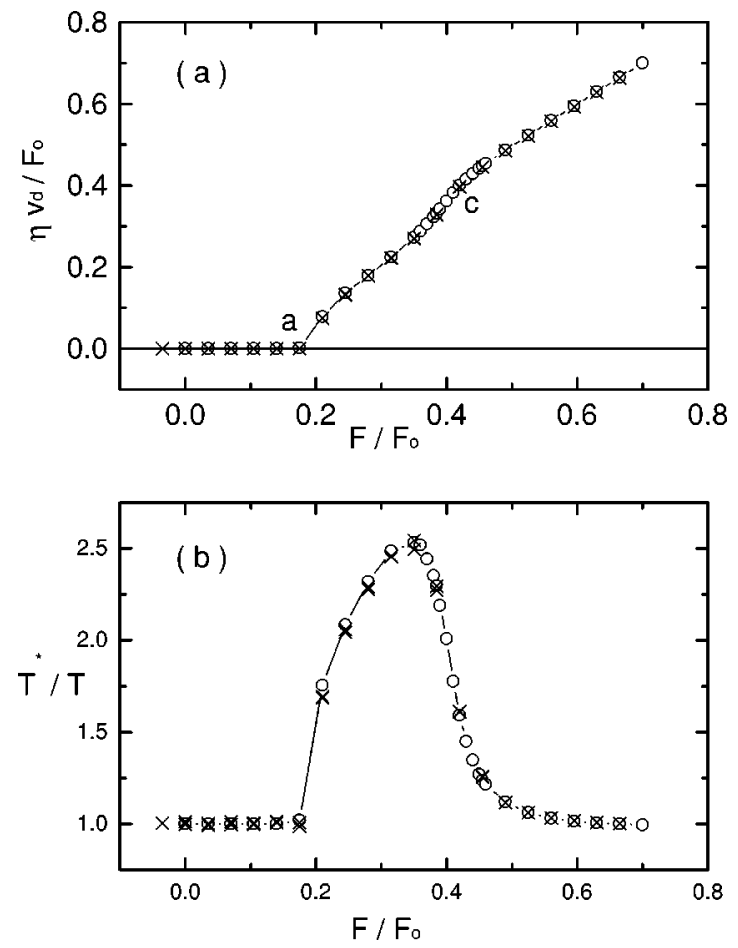

FIG. 6. Drift velocity $v_{d}$ and effective temperature $T^{*}$ as a function of external force $F$ for $\eta=0.6$ and $T=1.5$. Circles and crosses correspond to calculations using equilibration times differing by a factor of 10 .

can still be defined. In addition, a smooth sliding fluid to solid transition takes place at $F_{c}>F_{a}$. At this higher temperature, the system is able to relax in a short enough time to a stable single velocity, independent of the history of force variation. The behavior does not depend significantly on the rate of change of the force since calculations with different equilibration times give the same result as indicated in the figure. The hysteresis observed at low temperature is also not affected significantly. However, we cannot exclude that for large enough equilibration times the range of temperature where hysteresis is observed is much smaller. The behavior of $F_{a}, F_{b}$, and $F_{c}$ as a function of temperature is shown in Fig. 7. Within the present model, hysteresis disappears at $T$ $\sim U_{0}<T_{c}$ in contrast with the arguments ${ }^{2}$ suggesting that smooth sliding only occurs above $T_{c}$.

An important feature of the $F \times v_{d}$ curve in Fig. 4 is the

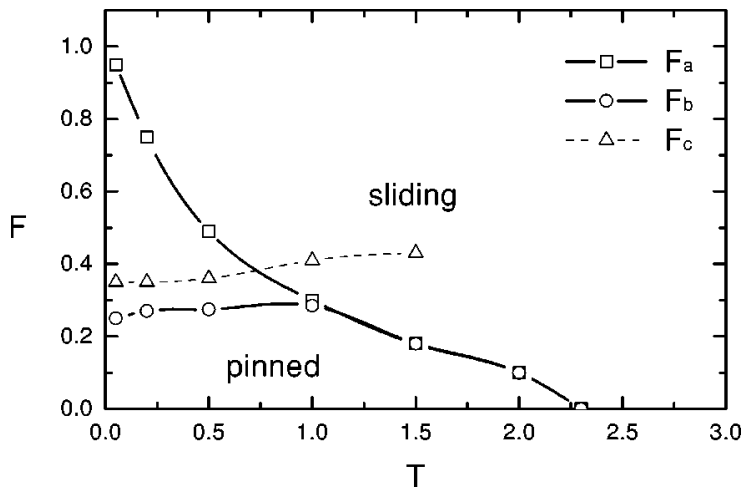

FIG. 7. Critical forces $F_{a}, F_{b}$, and $F_{c}$ as a function of temperature for $\eta=0.6$. 

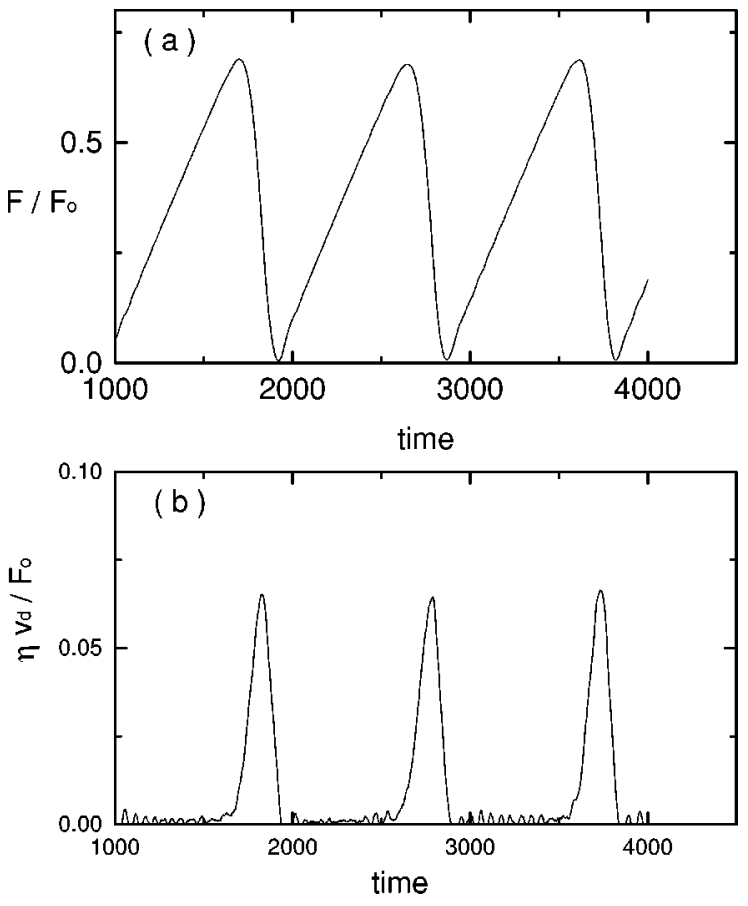

FIG. 8. Time evolution of the $v_{d}$ and $F$ when the system is pulled by a spring at constant velocity $v_{s}=0.01$, below $v_{b}$, for $\eta$ $=0.6, T=0.5$, and $K_{s}=0.5$.

existence of a "velocity gap," $0<v<v_{b}$, which leads to stick-and-slip motion. ${ }^{2}$ In the gap region, the overlayer cannot sustain a constant sliding velocity and stick-and-slip motion is expected when it is driven by a spring moving with constant velocity lying in the gap, as measured in sliding friction experiments. ${ }^{1}$ Smooth sliding is possible only for $v_{s}>v_{b}$. We have confirmed this behavior by calculating the time dependent drift velocity $v_{d}(t)$ and force $F(t)$ when each particle of the overlayer is subject to an elastic external force of the form

$$
F(t)=K_{s}\left(x_{c m}-v_{s} t\right)
$$

where $K_{s}$ is the spring elastic constant and $x_{c m}$ is the position of the center of mass of the overlayer. Indeed, stick and slip motion is obtained for $v_{s}<v_{b}$ as shown in Fig. 8 and smooth sliding motion for $v_{s}>v_{b}$ as shown in Fig. 9.

We have also studied the effects of varying the microscopic friction parameter $\eta$ on the nonlinear mobility. The $F \times v_{d}$ characteristics is shown in Fig. 10 for a larger value of $\eta=0.9$. In this case, the sliding fluid to solid transition occurs at $F_{c}>F_{a}$ and the return to the pinned solid phase at $F_{b}$ occurs at an effective temperature of the overlayer $T^{*}$ below $T_{c}$, so the results are qualitatively different from that for $\eta=0.6$. Persson ${ }^{2}$ has argued, based on hydrodynamical considerations that whenever $T^{*}<T_{c}$ is satisfied at $F_{b}$, the ratio $F_{b} / F_{a}$ between the kinetic and static friction forces should be a universal value $\sim 1 / 2$. For the present model, we find instead that the ratio $F_{b} / F_{a}$ depends on $\eta$ and $T$ as shown in Fig. 11. The ratio approaches 1 for $\eta \sim 3$ at $T$ $=0.5$. At lower temperatures, the results indicate that the ratio would asymptotically approach one at larger values of $\eta$. This behavior is expected since in the large $\eta$ limit the system is overdamped and hysteresis is not expected. In fact,
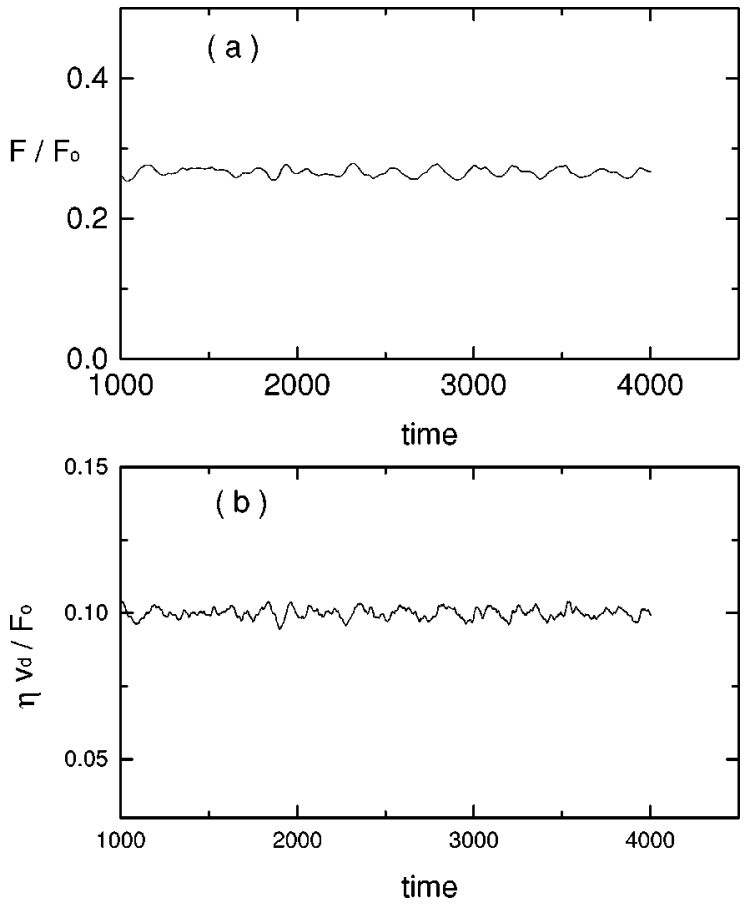

FIG. 9. Time evolution of the $v_{d}$ and $F$ when the system is pulled by a spring at constant velocity $v_{s}=0.1$, above $v_{b}$, for $\eta$ $=0.6, T=0.5$, and $K_{s}=0.5$.

we have confirmed through direct calculation that when the inertial term is dropped in the equation of motion, Eq. (3), hysteresis effects disappear and $F_{b} / F_{b}=1$ even for the lowest temperature in Fig. 11. This should be contrasted to the results obtained by Persson ${ }^{2}$ for the Lennard-Jones system where $F_{b} / F_{a}$ seems to saturate for large $\eta$ at $\approx 0.6$ for temperatures below $T_{c}$.
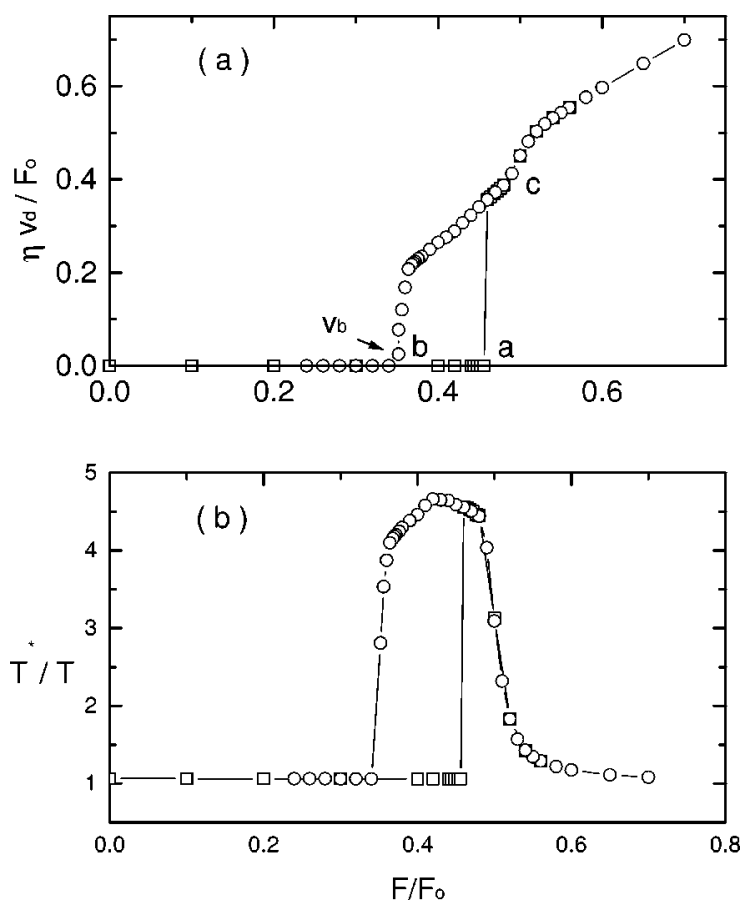

FIG. 10. Drift velocity $v_{d}$ and effective temperature $T^{*}$ as a function of external force $F$ for $\eta=0.9$ and $T=0.5$. Squares correspond to data for increasing $F$ and circles for decreasing $F$. The arrow indicates the minimum velocity $v_{b}$. 


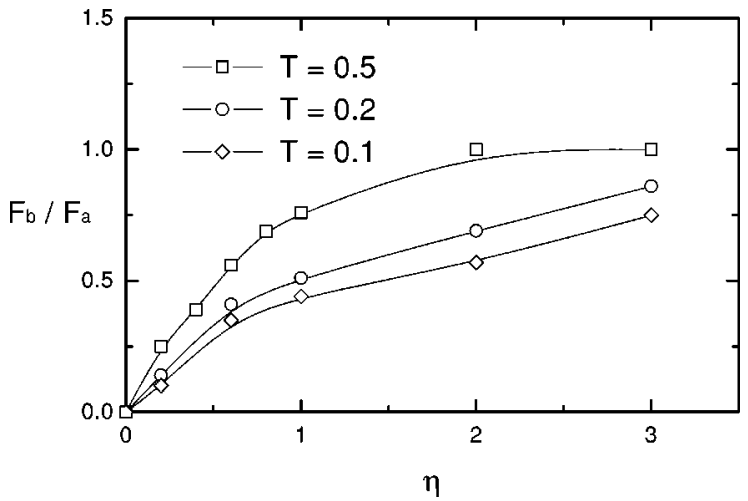

FIG. 11. Ratio $F_{b} / F_{a}$ as a function of $\eta$ and $T$.

We should note, however, that the nature of the hightemperature phase in the FK model is quite different from the Lennard-Jones system, with power law instead of the exponential decay of density correlations as a function of separation distance $r$. This follows from the fixed-neighbor constraint in the model of Eq. (1) that excludes topological defects, such as dislocations. It is well known that thermally excited dislocations can destroy crystalline order and lead to exponential decay of density correlations. ${ }^{14}$ Therefore, in the absence of dislocations, the equilibrium high temperature phase for $T>T_{c}$ and $F=0$ is a depinned elastic solid and correlations decay as a power law. In the sliding state for $F>0$, one thus expect that when the effective temperature of the overlayer $T^{*}$ is larger than $T$, overheating of the overlayer leads to power-law decay of density correlations, whereas long-range order should occur when $T^{*}=T$. This expected behavior is confirmed in Fig. 12 where the finitesize behavior of the structure factor peak for different values of $F$ and $\eta$ is shown, corresponding to the different dynamical phases in Figs. 4 and 10. For density correlations that decay algebraically as $r^{-} \eta_{o}$, the normalized peak height of $S(q)$ at the commensurate wave vector $q=\pi$ should scale as a power law $S(\pi) / N^{2} \propto N^{-q^{2} \eta_{o}}$ while it should be a constant when long-range order prevails. ${ }^{14}$ For the fluid phases where $T^{*}>T$, corresponding to the value of $F / F_{o}=0.32$ in Fig. 4 and $F / F_{o}=0.4$ in Fig. 10, the $\log -\log$ plot $S(\pi)$ vs $N$ has an apparent linear behavior with nonzero slope as shown in Fig. 12, consistent with algebraic order. For the pinned solid phase where $T^{*}=T$ at $F=0$, the quantity $S(\pi) / N^{2}$ is a constant indicating long-range order. For the sliding solid phase at $F / F_{o}=0.7$, the corresponding value for $S(\pi) / N^{2}$ decreases very slowly with increasing $N$, indicating that it is not a phase with full long-range order in contrast with the pinned solid phase at $F=0$ but much larger system sizes would be required to fully confirm this behavior.

The hydrodynamics arguments used by Persson ${ }^{2}$ suggesting a universal value $F_{b} / F_{a} \sim 0.5$ are based on the existence of a drag force acting on a nucleating domain of solid phase surrounded by the fluid phase. Since the nature of the fluid phases in the FK and Lennard-Jones models are very different, this argument is not valid for the FK model and this could account for the different behavior of the ratio $F_{b} / F_{a}$. However, in view of the many common features shared by the two models as shown in Figs. 4 and 10, it is also possible that the relation $F_{b} / F_{a} \sim 0.5$ is in fact valid only within a

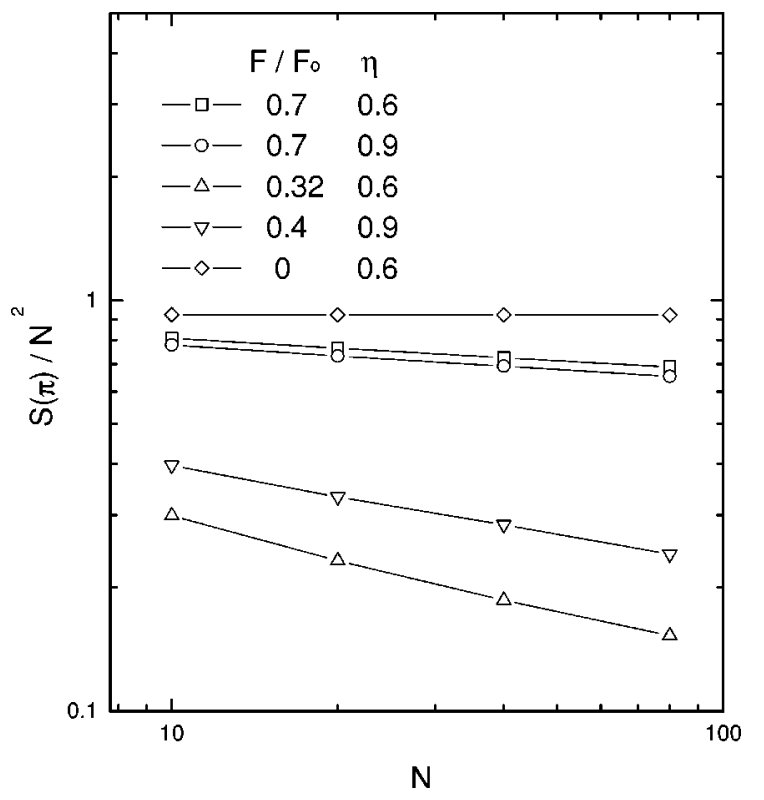

FIG. 12. Finite-size behavior of the peak in the structure factor $S(q)$ for different values of $F$ in Figs. 4 and 10.

limited range of $\eta$ and $T$, as shown in Fig. 11, and the Lennard-Jones system simply has a broader crossover region than the present model.

\section{CONCLUSIONS}

Comparing our results for the nonlinear response for the Frenkel-Kontorova model to the earlier study based on the Lennard-Jones interaction, ${ }^{2}$ we found that all the important features such as the appearance of dynamic sliding solid and fluid phases, the transition between these phases, the strong hysteresis effects, and the velocity gap for constant velocity sliding are common to both models. This indicates that these are robust features that do not depend on the details of the model such as many body interactions among the adatoms and surface geometry or anisotropies. The strong qualitative similarity of the results for mobility in these models to the effects observed in macroscopic sliding between two surfaces support strongly the idea that the response of the lubricant layer to an external force is the key factor in controlling boundary lubrication.

Besides the relevance to the macroscopic sliding friction, the dynamic phases generated by the external force are of intrinsic interest and have no counterparts in equilibrium. They are certainly worthy of more detailed study both experimentally and theoretically and may also be relevant to other types of driven lattice systems ${ }^{16}$ as for vortex lattices in type-II superconductors.

\section{ACKNOWLEDGMENTS}

This work was supported in part by a joint NSF-CNPq grant, an ONR grant (S.C.Y.), and by FAPESP (Proc. 97/ 07250-8) (E.G.). We thank M.R. Baldan for helpful discussions. 
${ }^{1}$ J. N. Israelachvili, Surf. Sci. Rep. 14, 109 (1992); H. Yoshizawa, P. McGuiggan, and J. Israelachvili, Science 259, 1305 (1993); B. Bhushan, J. N. Israelachvili, and U. Landman, Nature (London) 374, 607 (1995).

${ }^{2}$ B. N. J. Persson, Phys. Rev. Lett. 71, 1212 (1993); Phys. Rev. B 48, 18140 (1993); J. Chem. Phys. 103, 3849 (1995); Comments Condens. Matter Phys. 17, 281 (1995).

${ }^{3}$ Physics of Sliding Friction, edited by B. N. J. Persson and E. Tosatti (Kluwer, Dordrecht, 1996).

${ }^{4}$ B. N. J. Persson, Sliding Friction: Physical Principles and Applications (Springer, Heidelberg, 1998).

${ }^{5}$ E. Granato, M. R. Baldan, and S. C. Ying, in Physics of Sliding Friction, edited by B. N. J. Persson and E. Tosatti (Kluwer, Dordrecht, 1996), p. 103.

${ }^{6}$ Y. Braiman, F. Family, and H. G. E. Hentschel, Phys. Rev. E 53, R3005 (1996).

${ }^{7}$ O. M. Braun, T. Dauxois, M. V. Paliy, and M. Peyrard, Phys. Rev. Lett. 78, 1295 (1997); Phys. Rev. E 55, 3598 (1997).

${ }^{8}$ T. Kawaguchi and H. Matsukawa, Phys. Rev. B 56, 4261 (1997); 56, 13932 (1997).

${ }^{9}$ M. Weiss and F-J. Elmer, Z. Phys. B 104, 55 (1997).
${ }^{10}$ J. Krim, D. H. Solina, and R. Chiarello, Phys. Rev. Lett. 66, 181 (1991).

${ }^{11}$ Surface Diffusion: Atomistic and Collective Processes, edited by M. C. Tringides (Plenum, New York, 1998).

${ }^{12}$ K. Shinjo and M. Hirano, Surf. Sci. 283, 473 (1993).

${ }^{13}$ J. Frenkel and T. Kontorova, J. Phys. (Moscow) 1, 337 (1939); F. C. Frank and J. H. van der Merwe, Proc. R. Soc. London, Ser. A 198, 205 (1949).

${ }^{14}$ V. L. Pokrovsky and A. L. Talapov, Theory of Incommensurate Crystals, Soviet Scientific Reviews Vol. 1 (Harwood Academic, Glasgow, 1984).

${ }^{15}$ C. Carraro and D. R. Nelson, Phys. Rev. E 56, 797 (1997).

${ }^{16}$ P. Le Doussal and T. Giamarchi, Phys. Rev. B 57, 11356 (1998).

${ }^{17}$ M. P. Allen and D. J. Tildesley, Computer Simulation of Liquids (Oxford University Press, New York, 1993).

${ }^{18}$ Detailed calculations, however, are possible for the nearequilibrium dynamics in the overdamped limit by regarding Eq. (1) as the evolution equation of a growing interface of local heigh $x_{i j}$ with lattice effects represented by the pinning potential [P. Nozieres and F. Gallet, J. Phys. (Paris) 48, 353 (1987)]. 